\title{
Asociación entre las características morfológicas y la producción de leche en vacas Holstein
}

\author{
Piccardi, M.'; Balzarini, M. ${ }^{1,2}$; Bó, G.A. ${ }^{3,4}$; Capitaine Funes, A. ${ }^{5}$ \\ ${ }^{1}$ Fac.Cs.Agr.Univ.Nac.Córdoba, Av.Valparaíso s/n, Ciudad Universitaria, Córdoba, Argentina. Tel.:+54351- \\ 4334116 int: 216. ${ }^{2}$ Consejo Nacional de Investigaciones Científicas y Técnicas (CONICET). ${ }^{3}$ Instituto de \\ Reproducción Animal Córdoba (IRAC). ${ }^{4}$ Instituto de Ciencias Básicas, Carrera Med.Vet. Univ.Nac. \\ Villa María, Argentina. ${ }^{5}$ Dairytech SRL. E-mail: monicapiccardi@gmail.com
}

\begin{abstract}
Resumen
Piccardi, M.; Balzarini, M.; Bó, G.A.; Capitaine Funes, A.: Asociación entre las características morfológicas y la producción de leche en vacas Holstein. Rev. vet. 23: 2, 134-137, 2012. El objetivo de este estudio fue identificar las características morfológicas de tipo que contribuyen a explicar la variación de la producción en vacas Holstein. Se calificaron morfométricamente 1.290 vacas de la raza Holstein de cuatro tambos comerciales de la Provincia de Buenos Aires (Argentina). Los 15 caracteres morfológicos calificados fueron: inserción anterior de ubre (FU); altura de ubre posterior (RUH); ancho ubre posterior (RUW); ligamento suspensorio medio (UC); profundidad de la ubre (UD); colocación de pezones posterior (TP); longitud de los pezones (TL); estatura (ST); angulosidad (ANG); fortaleza (SG); ancho de grupa (RW); ángulo de grupa (RA); patas posteriores, vista posterior (RLRV); patas posteriores, vista lateral (RLS) y ángulo de pezuña (FA). El sistema de clasificación utilizado fue el propuesto por ACHA. Se aplicó el algoritmo de partición correspondiente a un árbol de clasificación sobre las dos categorías de producción de leche extremas (alta y baja producción) previamente definidas según los percentiles 33 y 66 de la variable 305 días equivalente maduro (305EM). Esta técnica multivariada constituye una alternativa a los modelos clásicos de regresión ya que sus resultados no se ven afectados por correlaciones entre las variables explicativas. Los resultados sugieren que valoraciones altas del ancho de ubre combinado con valoraciones altas de la fortaleza, son las métricas de tipo que más contribuyen a explicar mayores promedios de producción de leche en esta raza.
\end{abstract}

Palabras clave: bovino lechero, caracteres morfométricos, árbol de clasificación, colinealidad, tambos comerciales.

\begin{abstract}
Piccardi, M.; Balzarini, M.; Bó, G.A.; Capitaine Funes, A.: Morphological traits and the association with milk production in Holstein cows. Rev. vet. 23: 2, 134-137, 2012. The aim of this study was to identify the type traits that may help to explain the variation in milk production in Holstein cows. Animals were from four commercial dairies from Buenos Aires, Argentina $(n=1,290)$ that were morphometrically scored. The 15 morphological traits used were: fore udder attachment (FU), rear udder height (RUH), rear udder width (RUW), udder cleft (UC), udder depth (UD), teat placement (TP), teat length (TL), stature (ST), angularity (ANG), strength (GS), rump width (RW), rump angle (RA), feet and legs, rear view (RLRV), feet and legs, side view (RLS), and foot angle (FA). The qualification system used was proposed by ACHA (Asociación de Criadores de Holando Argentino). We applied the classification tree algorithm on the two categories of milk production (high and low production) that was previously defined by the percentiles 33 and 66 of the variable 305 days mature equivalent (305ME). The classification regression tree algorithm represents an alternative multivariate approach to the classical regression models. However, results were not affected by correlations between the variables. The results suggest that high scores of the trait rear udder width and high scores of the trait strength were associated with greatest milk production.
\end{abstract}

Key words: dairy cattle, morphometrical traits, classification tree, colinearity, commercial herds. 


\section{INTRODUCCIÓN}

Según la Asociación de Criadores de Holando Argentino (ACHA), la selección debe obtener vacas que no sólo produzcan mucha leche en una lactancia, sino que sean longevas; para ello deben ser sanas y parir regularmente ${ }^{1}$. La calificación y juzgamiento de animales evalúa rasgos de conformación buscando lograr ese objetivo de selección enunciado. El sistema de calificación es realizado por jurados entrenados, que evalúan visualmente 15 rasgos de conformación de cada animal, asignando puntaje a cada uno de ellos. Actualmente hay distintos sistemas de evaluación que son activamente usados para la selección de vacas. Varios autores han demostrado que estos caracteres morfológicos pueden ser usados como predictores de salud ${ }^{11,13 \text {, }}$ ${ }^{14}$, de fertilidad ${ }^{3,12,14}$, de longevidad ${ }^{6,15} \mathrm{y}$ de producción de leche ${ }^{3,5,8-10,16,17}$. El objetivo de este estudio fue identificar las características morfológicas de tipo que contribuyen a explicar la variación de la producción en vacas Holstein.

\section{MATERIAL Y MÉTODOS}

Se calificaron morfométricamente 1.290 vacas de la raza Holstein de 4 tambos comerciales ubicados en la Provincia de Buenos Aires (Argentina). Los 15 caracteres morfológicos calificados fueron: inserción anterior de ubre (FU); altura de ubre posterior (RUH); ancho ubre posterior (RUW); ligamento suspensorio medio (UC); profundidad de la ubre (UD); colocación de pezones posterior (TP); longitud de los pezones (TL); estatura (ST); angulosidad (ANG); fortaleza (SG); ancho de grupa (RW); ángulo de grupa (RA); patas posteriores, vista posterior (RLRV); patas posteriores, vista lateral (RLS) y ángulo de pezuña (FA). El sistema de calificación utilizado fue el propuesto por ACHA en su Manual de Juzgamiento de la raza Holando Argentino, en donde el score de los rasgos va de 10 a 100 puntos ${ }^{1}$.

Se categorizó a cada animal de acuerdo a la producción dada por el predictor 305EM: de baja producción si su valor era igual o inferior al percentil 33 (P33), que equivale a 7.570 litros y de alta producción si superaba el P66 de la población en estudio, equivalente a 8.726 litros. Luego se aplicó el algoritmo de partición correspondiente a un árbol de clasificación sobre las dos categorías de producción extremas previamente definidas ${ }^{4}$. Esta técnica multivariada constituye una alternativa a los modelos lineales clásicos de regresión ya que sus resultados no se ven afectados por interacciones o correlaciones entre variables regresoras (multicolinealidad), ni por la presencia de relaciones no lineales entre las variables regresoras y la cla- sificación realizada en función de la variable respuesta. Consiste en un conjunto de reglas determinadas por un procedimiento de ajuste mediante particiones binarias recursivas, donde el conjunto de datos es sucesivamente subdividido.

Esta técnica está relacionada con los conglomerados divisivos, en los que inicialmente todos los objetos son considerados como pertenecientes al mismo grupo. En cada instancia de separación el algoritmo analiza todas las variables regresoras y selecciona, para realizar la partición binaria de los datos, aquella regresora (y un umbral para esa regresora) que permite conformar los dos subgrupos o nodos de unidades más homogéneos dentro y más heterogéneos entre ellos que aquellos formados con cualquier otra variable. Los nodos formados se separarán nuevamente si cumplen una de las siguientes condiciones: si hay suficiente heterogeneidad para producir una partición de observaciones y/o el tamaño del nodo es superior al mínimo establecido para continuar el algoritmo. El proceso se detiene cuando alguna de estas condiciones no se cumple ${ }^{2}$. También se realizaron pruebas $t(\alpha=0,05)$ a los fines de analizar las significancias estadísticas de las diferencias en producción de leche entre los nodos del árbol de clasificación. El análisis estadístico fue realizado con el software InfoStat ${ }^{7}$.

\section{RESULTADOS Y DISCUSIÓN}

El árbol de clasificación (Figura 1, Tabla 1) sugiere que la variabilidad de los datos de producción de leche se debe en primer lugar al ancho de la ubre desde la vista posterior de la vaca (RUW), cual es un indicador de la capacidad de almacenar leche. Para esta variable se observó una clasificación umbral de 52,50, a partir del cual se separan los dos primeros nodos o grupos de vacas más diferentes. Las 351 vacas cuyo valor de RUW fue menor que el umbral, tuvieron mayoritariamente una producción por debajo del P33. Aún existe

Tabla 1. Producciones promedio (305EM) y probabilidades de tener producciones altas o bajas respecto al P33 (7.570 litros) y P66 (8.726 litros), clasificadas por características morfológicas en los dos primeros niveles de partición del árbol de clasificación.

\begin{tabular}{|c|c|c|c|c|c|}
\hline \multirow{2}{*}{ NP } & \multirow{2}{*}{$\begin{array}{l}\text { clasificación } \\
\text { según umbral }\end{array}$} & \multirow{2}{*}{$\begin{array}{l}\text { media } \\
305 \mathrm{EM}\end{array}$} & \multicolumn{2}{|c|}{$\begin{array}{c}\text { Probabilidad } \\
\text { (número de vacas) }\end{array}$} & \multirow{2}{*}{$\begin{array}{c}\mathrm{n} \\
\text { total }\end{array}$} \\
\hline & & & $\begin{array}{c}\text { producción } \\
\text { baja }\end{array}$ & $\begin{array}{c}\text { producción } \\
\text { alta }\end{array}$ & \\
\hline $1^{\circ}$ & RUW $(<=52,5)$ & $7.673,4 a$ & $0,63(222)$ & 0,37 (129) & 351 \\
\hline $2^{\circ}$ & RUW(>52,5) & $8.433,4 b$ & $0,40(206)$ & $0,60(309)$ & 515 \\
\hline $1^{\circ} 1$ & $\mathrm{FU}(<=72,5)$ & $7.638,8 \mathrm{a}$ & 0,65 (219) & $0,35(120)$ & 339 \\
\hline $1^{\circ} 2$ & $\mathrm{FU}(>72,5)$ & $8.655,5 b$ & $0,25(3)$ & $0,75(9)$ & 12 \\
\hline $2^{\circ} 1$ & $\mathrm{SG}(<=57,5)$ & $8.185,9 \mathrm{a}$ & $0,51(125)$ & $0,49(122)$ & 247 \\
\hline $2^{\circ} 2$ & $\mathrm{SG}(>57,5)$ & $8.662,4 b$ & $0,30(81)$ & $0,70(187)$ & 268 \\
\hline Total & & & 428 & 438 & 866 \\
\hline
\end{tabular}

NP: nivel de partición, RUW: ancho de la ubre, vista posterior; FU: inserción anterior de la ubre; SG: fortaleza. Letras distintas indican que existen diferencias estadísticamente significativas $(\mathrm{p} \leq 0,05)$. 
variabilidad de la producción que debe ser explicada; consecuentemente, el algoritmo continúa con la partición de los dos nodos formados anteriormente. En esta instancia de partición la variable que mejor explica la variabilidad remanente para el nodo de 351 es la inserción anterior de la ubre (FU) y para el nodo de 515, la fortaleza (SG). Del nodo de 351 vacas se desprenden dos nodos según la variable FU. Por debajo del valor de FU menor o igual a 72,50 se obtuvo mayoritariamente una producción inferior al P33 y por encima de ese umbral se obtuvieron mayoritariamente valores de producción de leche superiores al P66. Del nodo de 515 vacas (producción alta), 268 vacas con SG mayores a 57,50 tuvieron un rendimiento superior al P66 en mayor porcentaje (70\%) que las vacas con $\mathrm{SG}$ menor a 57,50 (49\%). Las producciones promedio de leche entre los grupos formados por el árbol de clasificación resultaron estadísticamente significativas $(\mathrm{p} \leq 0,05$; Tabla 1$)$. Varios autores han obtenido resultados similares en estudios que relacionan las características morfológicas de la ubre y los caracteres generales relacionados a la conformación del cuerpo con la producción ${ }^{3,5,8-10,16,17}$.

En conclusión, el ancho de la ubre posterior, la inserción anterior de la ubre y la fortaleza de la vaca resultaron, según el algoritmo del árbol de clasificación, los caracteres morfológicos de tipo de mayor importancia para clasificar vacas de baja y de alta producción. Valoraciones altas del ancho de ubre combinado con valoraciones altas del carácter morfológico de la fortaleza se asocian con los mayores promedios de producción de leche, mientras que valoraciones bajas en ancho de ubre combinada con valoraciones bajas de la inserción anterior de la ubre, se asocian a menores promedios de producción de leche.

\section{REFERENCIAS}

1. Asociación Argentina de Criadores de Holando Argentino (ACHA). 2012. On line: http://www.acha.org.ar/ index.php?option $=$ com_content $\&$ task $=$ view\&id $=205$ [revisado 20 de marzo de 2012].

2. Balzarini MG, González L, Tablada M, Casanoves F, Di Rienzo JA, Robledo CW. 2008. Manual del usuario, Ed.Brujas, Córdoba, Argentina, 333 p.

3. Berry DP, Buckley F, Dillon P, Evans RD, Veerkamp RF. 2004. Genetic relationships between linear type traits, milk yield, body weight, fertility and somatic cell count in primiparous dairy cows. Ir J Agric Food Res 43: 161-176.

4. Breiman L, Friedman JH, Olshen RA, Stone CJ. 1984. Classification and regression trees. Publ. Wadsworth International Group, Belmont (USA).

5. Corrales J, Cerón-Muñoz M, Cañas JA, Herrera C, Calvo S. 2011. Relationship between type traits and milk production in Holstein cows from Antioquia, Colombia. Revista MVZ (Córdoba, Colombia) 16: 2507-2513.

6. Daliri Z, Hafezian SH, Parvar A, Rahimi G. 2008. Genetic relationships among longevity, milk production and linear type traits in Iranian Holstein cattle. J Anim Vet Adv 7: $512-515$.

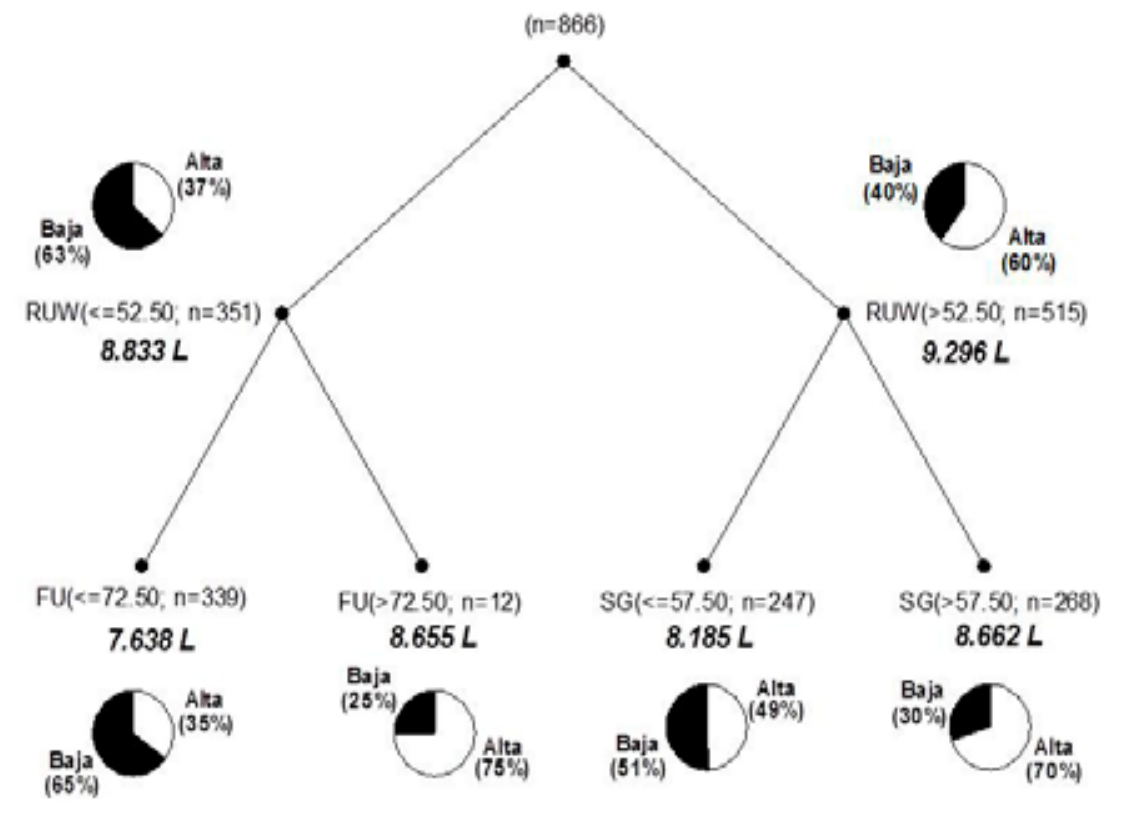

Figura 1. Árbol de clasificación con variable respuesta producción de leche categorizado según los percentiles 33 y 66 (P33 y P66). Se indica en cada nodo, explícita y gráficamente (gráficos de sectores) la probabilidad de tener rendimientos altos o bajos respecto a los P33 (7.570 litros) y P66 (8.726 litros). En cada nodo se indica la característica morfológica que el algoritmo tomó como criterio de partición, el valor umbral a partir del cual se separaron los nodos, y la cantidad de vacas (n) del nodo formado. 
7. Di Rienzo JA, Casanoves F, Balzarini MG, González L, Tablada M, Robledo CW. 2012. InfoStat versión 2012. Grupo InfoStat, FCA, Universidad Nacional de Córdoba, Argentina. URL http://www.infostat.com.ar.

8. Foster WW, Freeman AE, Berger PJ, Kuck A. 1989. Association of type traits scored linearity with production and herdlife of Holsteins. J Dairy Sci 72: 2651-2664.

9. Norman HD, Cassell BG, Pearson RE, Wiggans GR. 1981. Relation of first lactation production and conformation to lifetime performance and profitability in Jerseys. $J$ Dairy Sci 64:104-113.

10. Petersen ML, Hanson LB, Young CW, Miller KP. 1986. Conformation resulting from selection for milk yield of Holsteins. J Dairy Sci 69: 1884-1990.

11. Rogers GW, Hargrove GL, Lawlor TJ, Ebersole JL. 1991. Correlations among linear type traits and somatic cell counts. J Dairy Sci 74: 1087-1091.

12. Royal MD, Pryce JE, Woolliams JA, Flint AP. 2002. The genetic relationship between commencement of luteal activity and calving interval, body condition score, production, and linear type traits in Holstein-Friesian dairy cattle. J Dairy Sci 85: 3071-3080.
13. Rupp R, Boichard D. 1999. Genetic parameters for clinical mastitis, somatic cell score, production, udder type traits, and milking ease in first lactation Holsteins. J Dairy Sci 82: 2198-2204.

14. Pryce JE, Esslemont RJ, Thompson R, Veerkamp RF, Kossaibati MA, Simm G. 1998. Estimation of genetic parameters using health, fertility and production data from a management recording system for dairy cattle. Anim Sci 66: 577-584.

15. Samoré AB, Rizzi R, Rossoni A, Bagnato A. 2010. Genetic parameters for functional longevity, type traits, somatic cell scores, milk flow and production in the Italian Brown Swiss. Italian J Anim Sci 9: 145-152.

16. Tigges RJ, Pearson RE, Vinson WE. 1986. Prediction of lifetime relative net income from first lactation production and individual type traits in Holstein cows. J Dairy Sci 69: 204-210.

17. Van Doormaal BJ, Burnside EB, Schaeffer LR. 1986. An analysis of the relationships among stay ability production, and type in Canadian milk-recording programs. $J$ Dairy Sci 69: 510-517. 